\title{
Real-world safety of catheter ablation for atrial fibrillation with contact force or cryoballoon ablation
}

\author{
Andrea Natale ${ }^{1,2,3} \cdot$ Sanghamitra Mohanty ${ }^{1,4} \cdot$ Laura Goldstein $^{5} \cdot$ Tara Gomez $^{5} \cdot$ Tina D. Hunter $^{6}$ (D)
}

Received: 27 November 2019 / Accepted: 23 March 2020 / Published online: 11 May 2020

(C) The Author(s) 2020

\begin{abstract}
Purpose Real-world data can help medical administrators, physicians, and payers make evidence-based decisions regarding treatment choices. The objective of this study was to compare real-world safety outcomes with the latest catheter technologies used for the treatment of atrial fibrillation (AF).

Methods The Vizient Health Systems database, a large US hospital database, was used to compare acute complications in AF ablation with the contact force sensing THERMOCOOL SMARTTOUCH® Catheter or the THERMOCOOL SMARTTOUCH® SF Catheter (ST) versus the second-generation Arctic Front Advance ${ }^{\mathrm{TM}}$ Cryoablation Catheter (CB2) between September 2015 and June 2017. The primary outcome was a composite safety endpoint of acute ablation-related complications defined via ICD-10 diagnosis and procedure codes, including tamponade and other pericardial events, respiratory complications, stroke, cerebral or pre-cerebral occlusion/stenosis without infarction, vascular access complications, hemorrhage, phrenic nerve injury, myocardial infarction, and pulmonary embolism.

Results In total, 1473 ablations met all inclusion criteria (407 ST, 1066 CB2). Ablations for paroxysmal AF (PAF) had a lower complication rate than ablations for persistent AF (PsAF) (6.1\% vs. 7.3\%), as did ablations with ST compared with CB2 within each AF type (PAF 6.0\% vs. 6.1\%, PsAF 6.3\% vs. 7.8\%). Neither ablation catheter nor AF type was statistically significant after controlling for site volume, patient age, and comorbid conditions (ST vs. CB2: OR 0.86, $p=0.5544$; PsAF vs. PAF: OR 1.08, $p=$ $0.7376)$.

Conclusion Acute ablation-related complication rates were low and were not significantly associated with catheter technology. Increased risk of complication was attributable to low-volume sites and baseline patient characteristics.
\end{abstract}

Keywords Atrial fibrillation $\cdot$ Catheter ablation $\cdot$ Radiofrequency ablation $\cdot$ Contact force $\cdot$ Cryoablation $\cdot$ Safety

\section{Introduction}

The projected total cost of medical and indirect treatment for $\mathrm{AF}$ in 2019 is $\$ 35.7$ billion according to a 2016 report commissioned by the American Heart Association [1]. Both monetary and societal cost associated with AF can be minimized by utilizing the safest and most effective treatments, but

\author{
Tina D. Hunter \\ thunter@ctifacts.com \\ Andrea Natale \\ Andrea.Natale@stdavidsintl.com \\ Sanghamitra Mohanty \\ Mitra.Mohanty@stdavidsintl.com \\ Laura Goldstein \\ lgoldste@its.jnj.com \\ Tara Gomez \\ tgomez4@its.jnj.com
}

1 Texas Cardiac Arrhythmia Institute, St. David's Medical Center, Austin, TX, USA

2 Scripps Clinic Division Interventional Electrophysiology Address, San Diego, CA, USA

3 Metro Health Medical Center, Case Western Reserve University School of Medicine, Cleveland, OH, USA

4 Department of Internal Medicine, Dell Medical School, Austin, TX, USA

5 Health Economics and Market Access, Biosense Webster, Inc., Irvine, CA, USA

6 CTI Clinical Trial and Consulting Services, 100 East RiverCenter Boulevard, Covington, KY 41011, USA 
technological advances frequently outpace the evidence that is needed for informed decision-making.

Radiofrequency (RF) and cryoballoon (CB) ablations are well-established treatment modalities for $\mathrm{AF}$ ablation, and both are used frequently in paroxysmal (PAF) and persistent (PsAF) populations. Improvements in outcomes have been reported for both modalities with the introduction of the latest generations of each technology - contact force (CF) sensing $\mathrm{RF}$ ablation catheters and second-generation cryoballoon catheters [2-5]. Little published evidence exists currently that specifically compares the most recent products. Much of what does exist are single center studies that may not be representative and that lack sufficient power to detect potential differences in complication rates [6-10].

The objective of the current study was to compare acute procedure-related complications in a large population of realworld AF patients who underwent $\mathrm{AF}$ ablation with a contact force sensing THERMOCOOL SMARTTOUCH® Catheter or THERMOCOOL SMARTTOUCH® SF Catheter (ST; Biosense Webster, Irvine, CA) or a second-generation Arctic Front Advance ${ }^{\mathrm{TM}}$ Cryoablation Catheter (CB2; Medtronic, Minneapolis, $\mathrm{MN}$ ), representing clinical practices across the USA. The results of this study are intended to provide realworld evidence to help medical administrators, physicians, and payers make informed decisions regarding the latest catheter technologies.

\section{Methods}

\subsection{Study population}

This retrospective population-based study used the deidentified Vizient Health Systems database of real-world inpatient and hospital-based outpatient billing records, which include records for insured and uninsured healthcare recipients from over 400 hospitals in 42 states. Both inpatient and hospital-based outpatient visit information was submitted by 98\% of providers. This database includes patient demographics, hospital characteristics, complete diagnosis and procedure coding histories, and chargemaster billing records. All data used to perform this analysis were de-identified and accessed in compliance with the Health Insurance Portability and Accountability Act. As a retrospective analysis of a deidentified database, the research was exempt from IRB review under 45 CFR 46.101(b)(4).

Inclusion criteria for ablations comprising this study data were a procedure code for AF ablation coupled with a primary diagnosis of AF occurring between September 2015 and June 2017 in patients $\geq 18$ years old. Ablation cases that included a concomitant atrioventricular node ablation, valve replacement or repair, cardiac implant, surgical cardiac ablation, or surgical left atrial exclusion procedure were excluded. If a patient had multiple AF ablations meeting all inclusion and exclusion criteria to this point, only the first ablation was included. From within the population meeting these criteria, cohorts were defined based on catheter descriptions in the chargemaster billing records. Thus, ablation procedures that did not use an ST or CB2 catheter, or that had insufficient catheter descriptions to determine the type of ablation catheter used, were excluded. Finally, the population was restricted to patients for whom AF type was recorded so that the cohorts could be further divided into PAF and PsAF subgroups. The latter restriction was an important component of the study design because AF type is believed to potentially be associated with both risk level and catheter choice.

\subsection{Patient characteristics}

In addition to identifying the population with $\mathrm{AF}$ and the subgroups with PAF and PsAF, diagnosis codes were used to identify clinically important risk factors, including the presence of additional cardiac arrhythmias, risk factors comprising the $\mathrm{CHA}_{2} \mathrm{DS}_{2}-\mathrm{VASc}$ stroke risk score for AF patients, and available components of the HAS-BLED risk score for major bleeding. The $\mathrm{CHA}_{2} \mathrm{DS}_{2}-\mathrm{VASc}$ score was calculated by adding one point each for the presence of congestive heart failure (CHF), hypertension, age 65-74 years, diabetes mellitus, vascular disease, and female gender and two points each for history of stroke/transient ischemic attack (TIA) or age $\geq 75$.

\subsection{Ablation procedures and complications}

Ablation procedures using ST or $\mathrm{CB} 2$ catheters were identified via a text mining algorithm applied to the chargemaster descriptions and categories within the Vizient Health Systems real-world database. Ablations using both ST and CB2 catheters were assigned to the $\mathrm{CB} 2$ cohort in order to classify them based on the catheter most likely used for PV isolation.

The primary outcome was a composite endpoint of acute ablation-related complications recorded at any time during the ablation or prior to hospital discharge. These complications included tamponade and other pericardial events, respiratory complications, stroke, cerebral or pre-cerebral occlusion/ stenosis without infarction, vascular access complications, hemorrhage and/or blood transfusion, phrenic nerve injury, myocardial infarction (MI), and pulmonary embolism (PE).

All diagnoses and procedures used to define the inclusion and exclusion criteria, cohort classification, or characterization of patient risks were based on International Classification of Disease, 10th revision (ICD-10) diagnosis and procedure codes and Current Procedural Terminology (CPT) codes. 


\subsection{Statistical analysis}

Patient characteristics and complications were tabulated by catheter cohort within AF type, with means and standard deviations used to summarize continuous variables and counts and percentages to summarize categorical variables.

Logistic regression modeling was employed to determine whether the ablation catheter or type of AF was a significant predictor of the composite procedural complication outcome after adjusting for site procedure volume and any statistically significant differences in patient characteristics. The catheter cohort (ST versus $\mathrm{CB} 2$ ) and AF type (PAF versus PsAF) were included in the model regardless of statistical significance, while stepwise regression was used to select additional covariates with a statistical significance level of 0.05 . The patient characteristics considered for inclusion in the model included age, gender, payer type, presence of other arrhythmias (atrial flutter, supraventricular tachycardia, ventricular tachycardia), and comorbidities (cardiomyopathy, chronic kidney disease (CKD), CHF, chronic obstructive pulmonary disease (COPD), diabetes mellitus, history of stroke/TIA, hyperlipidemia, hypertension, obesity, obstructive sleep apnea, vascular disease). Analyses were performed using SAS version 9.4 (SAS Institute, Inc., Cary, NC, USA).

\section{Results}

\subsection{Study population}

There were 22,013 first AF ablations performed in adult patients within the study period of 2015-2017 that did not also include an exclusionary procedure. Of these ablations, 4620 used an ablation catheter that was identifiable in the data as ST or CB2. The final study population included the subset of 1473 ablations that also recorded the patient's AF type (1066 in the CB2 cohort and 407 in the ST cohort). The study ablations were contributed by 42 hospitals, with $18.3 \%$ performed in an inpatient setting.

The CB2 cohort had a lower percentage of PsAF patients than the ST cohort (38.3\% versus $47.2 \%$ ), so comparisons between cohorts were stratified by AF type (Table 1). The mean age across all patients at the time of their ablation was 63.9 years, which was slightly higher among PsAF patients than PAF patients and similar across catheter cohorts within each AF type. Males comprised $63.1 \%$ of the population overall, with more males in the PsAF group than the PAF group (71.2\% vs. $57.5 \%$ ) and in the ST cohort than the CB2 cohort within each AF group (PAF $63.3 \%$ vs. 55.6\%, PsAF $74.5 \%$ vs. $69.6 \%)$.

Comorbidity rates were higher on the whole in the PsAF group when compared with the PAF group, especially cardiomyopathy and CHF rates, which were approximately double in the PsAF group. The most prevalent concurrent arrhythmia was atrial flutter, which did not appear to impact catheter choice, as evidenced by similar rates across cohorts. However, a concurrent diagnosis of supraventricular tachycardia (SVT) did appear to impact catheter choice, as SVT rates were substantially higher in the ST cohort compared with CB2 cohort, both within the PAF and PsAF groups. The most prevalent of all comorbid conditions across the entire population was hyperlipidemia.

\subsection{Complications}

A total of 97 patients incurred a composite safety event, consisting of any one or more ablation-related complication(s) occurring during the ablation or prior to discharge (Table 2). The unadjusted composite complication rates were similar across catheter cohorts $(6.8 \% \mathrm{CB} 2,6.1 \% \mathrm{ST}, p=0.6723)$. In the PAF group, they were almost identical $(6.1 \%$ CB2, $6.0 \%$ ST, $p=0.9862$ ), while they were slightly higher in the CB2 cohort within the PsAF group, but this difference was not statistically significant (7.8\% CB2, 6.3\% ST, $p=0.4858$ ). Patients with complications were more likely to be inpatients than patients without complications $(49.5 \%$ vs. $16.1 \%)$, but the source data does not distinguish between patients that were admitted as inpatients prior to occurrence of a complication versus patients admitted as a consequence of a complication.

The number of ablations performed by a site had an impact on complication rates, with lower rates in sites that performed more ablations. After testing cutoffs of 10, 20, 30, and 40 procedures for statistical power, a high-volume site was defined as one with 20 or more ablations that met all inclusion and exclusion criteria for the study. Using this definition, 5.8\% of patients ablated at high-volume sites had an acute procedure-related complication versus $12.2 \%$ of patients ablated at low-volume sites $(p=0.0008)$ (Fig. 1). Complication rates did not vary significantly by catheter cohort within the high-volume sites $(5.9 \% \mathrm{CB} 2$ vs. $5.3 \% \mathrm{ST}, p=0.6904)$ or within the low-volume sites (13.4\% CB2 vs. $10.1 \%$ ST, $p=$ 0.5056).

After adjusting for site procedure volume and significant patient characteristics with logistic regression, neither the ablation catheter nor AF type had a significant impact on the occurrence of an acute procedure-related complication (Table 3). CKD and increasing age were the patient characteristics most strongly associated with an event, followed by COPD, CHF, obesity, and ventricular tachycardia, respectively.

\section{Discussion}

Complications following catheter ablation for AF adversely impact patient health, increase hospital costs, and negatively 
Table 1 Patient characteristics at the time of ablation

\begin{tabular}{|c|c|c|c|c|c|c|}
\hline \multirow[t]{2}{*}{ Characteristic } & \multicolumn{3}{|l|}{ PAF group } & \multicolumn{3}{|l|}{ PsAF group } \\
\hline & $\begin{array}{l}\mathrm{CB} 2 \\
(N=658) \\
n(\%)\end{array}$ & $\begin{array}{l}\mathrm{ST} \\
(N=215) \\
n(\%)\end{array}$ & $P$ value & $\begin{array}{l}\text { CB2 } \\
(N=408) \\
n(\%)\end{array}$ & $\begin{array}{l}\mathrm{ST} \\
(N=192) \\
n(\%)\end{array}$ & $P$ value \\
\hline \multicolumn{7}{|l|}{ Demographics } \\
\hline Age, years (mean, SD) & $63.2,10.6$ & $62.7,12.1$ & 0.5263 & $65.3,10.0$ & $64.7,9.6$ & 0.5100 \\
\hline Male & $366(55.6)$ & $136(63.3)$ & 0.0494 & $284(69.6)$ & $143(74.5)$ & 0.2192 \\
\hline $\mathrm{CHA}_{2} \mathrm{DS}_{2}$-VASC score & & & 0.1066 & & & 0.6021 \\
\hline 0 & $116(17.6)$ & $47(21.9)$ & & $60(14.7)$ & $31(16.1)$ & \\
\hline 1 & $164(24.9)$ & $61(28.4)$ & & $82(20.1)$ & $52(27.1)$ & \\
\hline 2 & 177 (26.9) & $43(20.0)$ & & $107(26.2)$ & $46(24.0)$ & \\
\hline 3 & $98(14.9)$ & $28(13.0)$ & & $80(19.6)$ & $34(17.7)$ & \\
\hline 4 & $53(8.1)$ & $17(7.9)$ & & $40(9.8)$ & $16(8.3)$ & \\
\hline 5 & $32(4.9)$ & $14(6.5)$ & & $22(5.4)$ & $7(3.6)$ & \\
\hline 6 & $14(2.1)$ & $3(1.4)$ & & $13(3.2)$ & $3(1.6)$ & \\
\hline 7 & $3(0.5)$ & $0(0.0)$ & & $3(0.7)$ & $2(1.0)$ & \\
\hline 8 & $0(0.0)$ & $2(0.9)$ & & $1(0.2)$ & $1(0.5)$ & \\
\hline 9 & $1(0.2)$ & $0(0.0)$ & & $0(0.0)$ & $0(0.0)$ & \\
\hline \multicolumn{7}{|l|}{ Other types of arrhythmia } \\
\hline Atrial flutter & 188 (28.6) & $62(28.8)$ & 0.9403 & $111(27.2)$ & $47(24.5)$ & 0.4793 \\
\hline Supraventricular tachycardia (SVT) & $36(5.5)$ & $32(14.9)$ & $<.0001$ & $27(6.6)$ & $20(10.4)$ & 0.1062 \\
\hline Ventricular tachycardia & $8(1.2)$ & $5(2.3)$ & 0.2434 & $7(1.7)$ & $3(1.6)$ & 0.8912 \\
\hline \multicolumn{7}{|l|}{ Comorbidities } \\
\hline Cardiomyopathy & $79(12.0)$ & $20(9.3)$ & 0.2777 & $97(23.8)$ & $39(20.3)$ & 0.3447 \\
\hline Chronic kidney disease (CKD) & $44(6.7)$ & $20(9.3)$ & 0.2015 & $30(7.4)$ & $13(6.8)$ & 0.7965 \\
\hline Congestive heart failure (CHF) & $121(18.4)$ & $35(16.3)$ & 0.4832 & $141(34.6)$ & $55(28.6)$ & 0.1497 \\
\hline Chronic obstructive pulmonary disease (COPD) & $105(16.0)$ & $28(13.0)$ & 0.2986 & $76(18.6)$ & $25(13.0)$ & 0.0869 \\
\hline Diabetes Mellitus & $133(20.2)$ & $31(14.4)$ & 0.0590 & $104(25.5)$ & $38(19.8)$ & 0.1255 \\
\hline History of Stroke/TIA & $44(6.7)$ & $18(8.4)$ & 0.4036 & $31(7.6)$ & $14(7.3)$ & 0.8943 \\
\hline Hyperlipidemia & $332(50.5)$ & $96(44.7)$ & 0.1394 & $219(53.7)$ & $104(54.2)$ & 0.9105 \\
\hline Hypertension & $73(11.1)$ & $23(10.7)$ & 0.8718 & $54(13.2)$ & $26(13.5)$ & 0.9180 \\
\hline Obesity & $160(24.3)$ & $43(20.0)$ & 0.1934 & $111(27.2)$ & $36(18.8)$ & 0.0247 \\
\hline Obstructive sleep apnea & $166(25.2)$ & $45(20.9)$ & 0.2013 & $130(31.9)$ & $52(27.1)$ & 0.2348 \\
\hline Vascular disease & $173(26.3)$ & $57(26.5)$ & 0.9493 & $126(30.9)$ & $54(28.1)$ & 0.4918 \\
\hline
\end{tabular}

influence patient satisfaction and quality of life. As a result, it is important to understand the impact, if any, that the latest technologies and ablation methodologies will have on the incidence of complications. It is also important to understand which patients will be at the greatest risk of complication. In the current study, patients undergoing AF ablation with two of the latest catheter technologies had similarly low rates of acute ablation-related complications (ST 6.1\%, CB2: 6.8\%, $p=$ $0.6723)$. After adjusting for site volume and patient characteristics in a logistic regression model, neither the catheter nor the type of AF was statistically significantly associated with the occurrence of a complication. The most predictive patient characteristics, in order of association with complications, were CKD, age, COPD, CHF, obesity, and ventricular tachycardia. Sites that performed a higher volume of ablations also had significantly lower complication rates than sites with lower volumes.

In the nationally representative National Inpatient Sample (NIS) database, comprising 93,801 AF procedures performed between 2000 and 2010, the incidence of AF ablation-related complications was $6.5 \%$ [11]. The current study found a similar incidence of complications in both the ST and CB2 catheter cohorts, with $6.1 \%$ and $6.8 \%$ respectively. The most common complications in the NIS database study were combined cardiac events $(2.5 \%)$, followed by vascular events $(1.5 \%)$, respiratory events $(1.3 \%)$, and neurologic events $(1.0 \%)$, which is roughly similar to the event categories and rates reported in the current study. It should be noted that the NIS 
Table 2 Procedure-related complications occurring prior to ablation discharge

\begin{tabular}{|c|c|c|c|c|c|c|}
\hline \multirow[t]{2}{*}{ Complication } & \multicolumn{3}{|c|}{ PAF group } & \multicolumn{3}{|c|}{ PsAF group } \\
\hline & $\begin{array}{l}\mathrm{CB} 2 \\
N=658 \\
n(\%)\end{array}$ & $\begin{array}{l}\mathrm{ST} \\
N=215 \\
n(\%)\end{array}$ & $\begin{array}{l}P \\
\text { value }\end{array}$ & $\begin{array}{l}\text { CB2 } \\
N=408 \\
n(\%)\end{array}$ & $\begin{array}{l}\mathrm{ST} \\
N=192 \\
n(\%)\end{array}$ & $\begin{array}{l}P \\
\text { value }\end{array}$ \\
\hline Composite complication outcome & $40(6.1)$ & $13(6.0)$ & 0.9862 & $32(7.8)$ & $12(6.3)$ & 0.4850 \\
\hline \multicolumn{7}{|l|}{ Individual complications } \\
\hline Tamponade/pericardial events & $12(1.8)$ & $4(1.9)$ & 0.9722 & $9(2.2)$ & $2(1.0)$ & 0.3214 \\
\hline Pericardial drainage procedure & $4(0.6)$ & $0(0.0)$ & 0.2519 & $2(0.5)$ & $1(0.5)$ & 0.9604 \\
\hline Respiratory complications & $15(2.3)$ & $0(0.0)$ & 0.0255 & $7(1.7)$ & $4(2.1)$ & 0.7542 \\
\hline $\begin{array}{l}\text { Stroke or other cerebral/pre-cerebral } \\
\text { occlusion/stenosis }\end{array}$ & $10(1.5)$ & $3(1.4)$ & 0.8960 & $6(1.5)$ & $3(1.6)$ & 0.9311 \\
\hline Vascular access events & $5(0.8)$ & $5(2.3)$ & 0.0611 & $5(1.2)$ & $3(1.6)$ & 0.7371 \\
\hline Hemorrhage/blood transfusion & $8(1.2)$ & $2(0.9)$ & 0.7326 & $3(0.7)$ & $2(1.0)$ & 0.7002 \\
\hline Phrenic nerve complication & $2(0.3)$ & $0(0.0)$ & 0.4183 & $2(0.5)$ & $0(0.0)$ & 0.3312 \\
\hline Myocardial infarction & $1(0.2)$ & $1(0.5)$ & 0.4044 & $1(0.2)$ & $0(0.0)$ & 0.4924 \\
\hline Pulmonary embolism & $1(0.2)$ & $0(0.0)$ & 0.5674 & $0(0.0)$ & $1(0.5)$ & 0.1446 \\
\hline
\end{tabular}

study included a $0.46 \%$ death rate for patients ablated in an inpatient setting. While our data did not allow identification of in-hospital death due to patient de-identification algorithms used by the data vendor, we would expect deaths to have a much smaller impact on our rates, due to the fact that only $18.3 \%$ of our ablations were inpatient procedures and also due to the later timeframe of our study and the improvement in the safety of AF ablation in recent years. Complications in the NIS study were associated with physicians who performed $<25$ ablations annually and hospital volumes of $<40$ procedures annually, which parallels our finding that a site procedure volume of at least 20 ablations meeting our inclusion

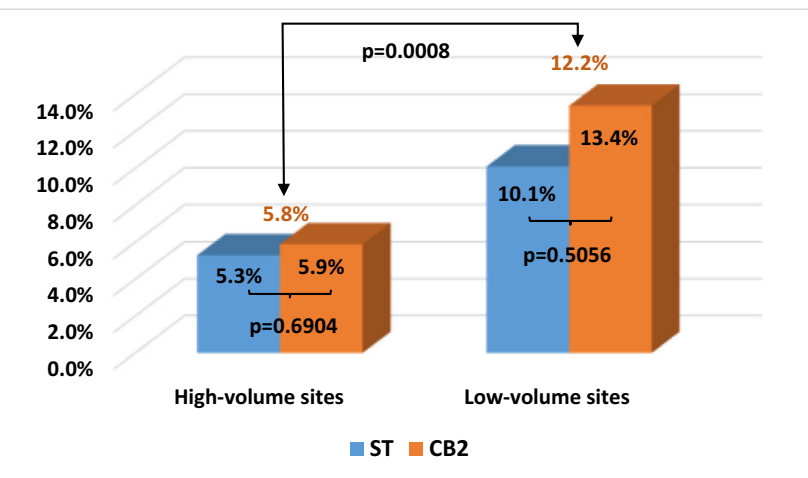

Fig. 1 Acute complications across high- and low-volume centers. A total of $5.8 \%$ of patients ablated at high-volume sites had an acute procedurerelated complication versus $12.2 \%$ of patients ablated at low-volume sites $(p=0.0008)$. Complication rates did not vary significantly by catheter cohort within the high-volume sites $(5.9 \% \mathrm{CB} 2$ vs. $5.3 \% \mathrm{ST}, p=$ $0.6904)$ or within the low-volume sites $(13.4 \%$ CB2 vs. $10.1 \%$ ST, $p=$ $0.5056)$ criteria was associated with a significant reduction in complication rates.

Another study used the Premier Healthcare Database, a US hospital database similar to the one used in the current study, to compare $1261 \mathrm{RF}$ ablations with $1276 \mathrm{CB} 2$ ablations [12]. Though focused on cost, the study also reported similar complication rates in RF and CB2 cohorts after adjustment for significant patient and hospital characteristics ( $p$ values, 0.4888 and 0.5072 for ablations performed in the inpatient and outpatient settings). The complication endpoint in this study was constructed very similarly to the one used in the current study. However, the prior study included ablations performed in 2013-2014; thus, the RF cohort would have been comprised almost exclusively of non-CF catheters due to the 2014 FDA approval of ST.

Procedure-related complications for ST and CB2 ablations have been reported in several prospective interventional studies. The first to specifically compare these particular catheters is currently underway and recently reported on the initial results, including periprocedural complication rates $[13,14]$. As in the current study, the ST cohort had a non-significantly lower rate of events than the two CB2 cohorts $(2.6 \%$ vs. $5.2 \%$ and $6.0 \%, p=0.24)$. In addition, the prospective multicenter SMART-AF trial enrolled 161 PAF patients in a single ST ablation cohort, reporting $2.5 \%$ tamponade, $1.9 \%$ pericarditis, and $2.5 \%$ vascular complication rates within 7 days of the procedure [15]. There were no cases of atrioesophageal fistula, PV stenosis, thromboembolism, cerebrovascular accident, MI, stroke, or death during the 12 months following the procedure. Among 762 patients randomized to RF or cryoballoon ablation in the FIRE and ICE study, the primary composite safety endpoint was not significantly different through 12 months (HR, 
Table 3 Logistic regression model of composite acute ablation-related complication

\begin{tabular}{lll}
\hline Patient characteristic & $\begin{array}{l}\text { Odds ratio } \\
{[95 \% \text { confidence interval }]}\end{array}$ & $P$ value \\
\hline Primary Predictors of Interest & & 0.5544 \\
Type of catheter ablation: ST versus CB2 & $0.86[0.53,1.41]$ & 0.7376 \\
Type of AF: PsAF versus PAF & $1.08[0.70,1.67]$ & \\
Additional Predictors with Significance at 0.05 Level & & 0.0003 \\
Chronic kidney disease (CKD) & $2.81[1.59,4.94]$ & 0.0019 \\
Age, per year of increase & $1.04[1.01,1.06]$ & 0.0024 \\
Ablation at site with $\geq 20$ ablations in study & $0.45[0.26,0.75]$ & 0.0153 \\
Chronic obstructive pulmonary disease (COPD) & $1.83[1.12,2.98]$ & 0.0260 \\
Congestive heart failure (CHF) & $1.70[1.07,2.70]$ & 0.0370 \\
Obesity & $1.66[1.03,2.66]$ & 0.0529 \\
Ventricular tachycardia & $2.99[0.99,9.08]$ & \\
\hline
\end{tabular}

$0.78,95 \% \mathrm{CI},(0.52,1.18))[16]$. However, this trial had an imbalance of $75.6 \% \mathrm{CB} 2$ catheters (vs. first generation $\mathrm{CB}$ ) compared to only $24.7 \% \mathrm{CF}$ catheters (vs. non-CF RF). Groin complications (1.0\% CB, 2.1\% RF) and phrenic nerve injury ( $1.3 \%$ in $\mathrm{CB}$ only) were the most common complications in this study, with no atrioesophageal fistula, pulmonary vein stenosis, or procedure-related death.

Additional non-randomized clinical studies, primarily utilizing smaller samples of consecutive ablations performed at a single site, have also compared ST vs. CB2 [6-8, 17-19]. None of these studies found a statistically significant difference in complication rates between catheter cohorts, though none were powered to do so. Several studies did report that the profiles of events differed in some respect. In particular, three studies reported that phrenic nerve palsy was exclusively seen in the CB2 arms $[6,17,19]$, while two reported that tamponade was only seen in the ST arms [6, 19]. Another three of the studies reported less fluoroscopy exposure in the ST arms [7, 8, 18], which is a safety consideration that could not be studied in the current hospital database analysis due to the lack of procedural detail reported in administrative databases.

While complication rates are commonly reported in studies of AF ablation, the patient characteristics associated with risk of experiencing an ablation-related complication are less studied. The current study was comprised of 1473 ablations, 97 of which resulted in complications. This sample size was sufficient to identify 6 baseline patient characteristics that were significantly associated with complications at a level of approximately 0.05 or below. Despite some differences in the specific characteristics that were measured across the two studies, both the current study and a 2016 study by Padala et al. identified CHF, CKD/renal disease, COPD, and age as important risk factors for acute ablation-related complications [20]. It is well-known that CHF and age are among the greatest risk factors for stroke in an $\mathrm{AF}$ population, as commonly measured via the $\mathrm{CHA}_{2} \mathrm{DS}_{2}$-VASc risk score, [21] and similarly that age and CKD increase bleeding risk, as per the HAS-BLED risk score [22, 23]. Presumably, COPD would increase the risk of respiratory complications.

\subsection{Limitations}

The primary limitations of this study are the retrospective design and the use of administrative hospital data, which does not provide the level of clinical detail afforded by prospectively designed clinical studies. Patients could not be followed beyond their ablation discharge, thus limiting the capture of late complications or clinical effectiveness. In addition, the use of administrative hospital data did not allow inclusion of procedural details such as fluoroscopy usage, complete preablation medical and medication histories, death, or clinical details such as the severity of comorbidities. These unknowns could potentially confound or bias the results. However, the baseline characteristics that were captured at the time of the procedure and are known to be risk factors in an AF population were similar across catheter cohorts. Another unmeasured patient characteristic that could impact safety outcomes is oral anticoagulation (OAC) strategy. However, if OAC is used per the most recent guidelines, it would not be expected to differ across cohorts. Moreover, our comparison only requires that the overall population ablated with ST is not systemically anticoagulated differently than the overall population ablated with cryoablation, which we believe is a very reasonable assumption.

Another limitation of any retrospective study design is that the sample size is dictated by the data that is available from the source that meets all inclusion criteria. Thus, the sample size is not chosen prospectively to correspond to the statistical power required for testing of hypotheses. Without statistically powered hypotheses, a lack of statistical significance (i.e., high $p$ values) does not imply a lack of clinical significance. Therefore, any observed differences in effect sizes that are not 
statistically significant but appear to be of clinically significant magnitude will require further studies to confirm.

Text mining algorithms used to define the catheter cohorts were limited to hospitals that recorded a sufficient description of the ablation catheter, resulting in exclusion of ablations that may have otherwise met the inclusion criteria. In particular, in many cases, it was impossible to distinguish among manufacturers or generations of RF catheters, likely resulting in a more complete capture of the CB2 cohort versus an underrepresentation of the ST cohort. Also, the ST cohort included two generations of contact force catheter because the data available at the start of the study did not yet include sufficient quantity of ablations using the newer porous cooling tip design. Thus, any safety benefits attributable to the new cooling tip design could not be studied.

Despite the limitations of the retrospective study design and the inherent shortcomings of administrative data, the current study has several unique strengths. First and foremost, the large and nationally representative population enabled us to present a highly generalizable picture of current clinical practice in the USA. Unlike most clinical trials or single-site studies of consecutive patients, this data reflects a combination of high-volume and low-volume sites and thus is more reflective of the full spectrum of AF ablation treatment in the USA. The importance of this site variation is reflected in the finding that sites performing low volumes of ablations had complication rates more than double the rates seen in sites performing higher volumes. In addition to the benefits of a diverse population, the large sample size allowed for stratification of complications by both catheter and AF type and also for identification of patient characteristics that are associated with a higher risk of experiencing an acute ablation-related complication.

\section{Conclusions}

This large real-world study found a slightly lower but not statistically different rate of complications in AF ablations performed with ST compared with CB2. Site volume was an important predictor of complication rates, as was patient age and several baseline comorbid conditions. AF type was not a significant predictor after adjusting for site volume and patient characteristics. This evidence fills a gap in the understanding of ablation-related complication rates with the latest ablation technologies as well as a comparison between the latest generations of RF and cryoballoon catheter technologies for $\mathrm{AF}$ ablation in a large real-world US population.

Acknowledgments The authors appreciate the input of Rebecca Beals and Tom Wei for their assistance in the study and manuscript development process.
Funding information Analysis and report writing were funded by Biosense Webster, Inc.

The study was funded by BWI, and BWI played a critical role in study design, analysis interpretation of data, as well as writing the report.

\section{Compliance with ethical standards}

Conflict of interest A Natale is a paid consultant for Biosense Webster, Inc., Medtronic, Biotronik, St. Jude/Abbott, Boston Scientific, and Baylis. Neither honoraria nor payments were made for authorship. S Mohanty has no disclosures. L Goldstein and T Gomez are employees of Biosense Webster, Inc., a Johnson and Johnson company. TD Hunter is an employee of CTI Clinical Trial and Consulting Services, which is a consultant to Biosense Webster, Inc., the study sponsor.

Open Access This article is licensed under a Creative Commons Attribution 4.0 International License, which permits use, sharing, adaptation, distribution and reproduction in any medium or format, as long as you give appropriate credit to the original author(s) and the source, provide a link to the Creative Commons licence, and indicate if changes were made. The images or other third party material in this article are included in the article's Creative Commons licence, unless indicated otherwise in a credit line to the material. If material is not included in the article's Creative Commons licence and your intended use is not permitted by statutory regulation or exceeds the permitted use, you will need to obtain permission directly from the copyright holder. To view a copy of this licence, visit http://creativecommons.org/licenses/by/4.0/.

\section{References}

1. Khavjou O, Phelps D, Leib A \& RTI International. Projections of cardiovascular disease prevalence and costs: 2015-2035. Technical report prepared for the American Heart Association; 2016. https:// www.heart.org/idc/groups/heart-public/@wcm/@adv/documents/ downloadable/ucm_491513.pdf. Accessed Aug 2018.

2. Nakamura K, Naito S, Sasaki T, Nakano M, Minami K, Nakatani Y, et al. Randomized comparison of contact force-guided versus conventional circumferential pulmonary vein isolation of atrial fibrillation: prevalence, characteristics, and predictors of electrical reconnections and clinical outcomes. J Interv Card Electrophysiol. 2015;44(3):235-45. https://doi.org/10.1007/s10840-015-0056-7.

3. Kimura M, Sasaki S, Owada S, Horiuchi D, Sasaki K, Itoh T, et al. Comparison of lesion formation between contact force-guided and non-guided circumferential pulmonary vein isolation: a prospective, randomized study. Heart Rhythm. 2014;11(6):984-91. https://doi.org/10.1016/j.hrthm.2014.03.019.

4. Pandya B, Sheikh A, Spagnola J, Bekheit S, Lafferty J, Kowalski M. Safety and efficacy of second-generation versus first-generation cryoballoons for treatment of atrial fibrillation: a meta-analysis of current evidence. J Interv Card Electrophysiol. 2016;45(1):49-56. https://doi.org/10.1007/s10840-015-0075-4.

5. Conti S, Moltrasio M, Fassini G, Tundo F, Riva S, Dello Russo A, et al. Comparison between first- and second-generation cryoballoon for paroxysmal atrial fibrillation ablation. Cardiol Res Pract. 2016;2016:5106127. https://doi.org/10.1155/2016/5106127.

6. Kardos A, Kis Z, Som Z, Nagy Z, Foldesi C. Two-year follow-up after contact force sensing radiofrequency catheter and secondgeneration cryoballoon ablation for paroxysmal atrial fibrillation: a comparative single Centre study. Biomed Res Int. 2016;2016: 6495753. https://doi.org/10.1155/2016/6495753.

7. Jourda F, Providencia R, Marijon E, Bouzeman A, Hireche H, Khoueiry Z, et al. Contact-force guided radiofrequency vs. 
second-generation balloon cryotherapy for pulmonary vein isolation in patients with paroxysmal atrial fibrillation-a prospective evaluation. Europace. 2015;17(2):225-31. https://doi.org/10.1093/ europace/euu215.

8. Matta M, Anselmino M, Ferraris F, Scaglione M, Gaita F. Cryoballoon vs. radiofrequency contact force ablation for paroxysmal atrial fibrillation: a propensity score analysis. J Cardiovasc Med (Hagerstown). 2018;19(4):141-7. https://doi.org/10.2459/ JCM.0000000000000633.

9. Ciconte G, Baltogiannis G, de Asmundis C, Sieira J, Conte G, Di Giovanni G, et al. Circumferential pulmonary vein isolation as index procedure for persistent atrial fibrillation: a comparison between radiofrequency catheter ablation and second-generation cryoballoon ablation. Europace. 2015;17(4):559-65. https://doi. org/10.1093/europace/euu350.

10. Ciconte G, Velagic V, Mugnai G, Saitoh Y, Irfan G, Hunuk B, et al. Electrophysiological findings following pulmonary vein isolation using radiofrequency catheter guided by contact-force and secondgeneration cryoballoon: lessons from repeat ablation procedures. Europace. 2016;18(1):71-7. https://doi.org/10.1093/europace/ euv224.

11. Deshmukh A, Patel NJ, Pant S, Shah N, Chothani A, Mehta K, et al. In-hospital complications associated with catheter ablation of atrial fibrillation in the United States between 2000 and 2010: analysis of 93801 procedures. Circulation. 2013;128(19):2104-12. https://doi. org/10.1161/CIRCULATIONAHA.113.003862.

12. Hunter TD, Palli SR, Rizzo JA. Cost comparison of radiofrequency catheter ablation versus cryoablation for atrial fibrillation in hospitals using both technologies. J Med Econ. 2016;19(10):959-64. https://doi.org/10.1080/13696998.2016.1187153.

13. Andrade JG, Deyell MW, Badra M, Champagne J, Dubuc M, Leong-Sit $\mathrm{P}$, et al. Randomised clinical trial of cryoballoon versus irrigated radio frequency catheter ablation for atrial fibrillation-the effect of double short versus standard exposure cryoablation duration during pulmonary vein isolation (CIRCA-DOSE): methods and rationale. BMJ Open. 2017;7(10):e017970. https://doi.org/10. 1136/bmjopen-2017-017970.

14. Andrade J, Champagne J, Dubuc M, Deyell M, Verma A, Macle L, et al. A randomized clinical trial of cryoballoon vs irrigated radiofrequency catheter ablation for atrial fibrillation: the CIRCA-DOSE study. Presented at The Annual Congress of the European Heart Rhythm Association; 2019. https://www.escardio.org/Congresses\&-Events/EHRA-Congress/Congress-resources/News/the-latestfindings-in-atrial-fibrillation-circa-dose. Accessed Aug 2018.

15. Natale A, Reddy VY, Monir G, Wilber DJ, Lindsay BD, McElderry HT, et al. Paroxysmal AF catheter ablation with a contact force sensing catheter: results of the prospective, multicenter SMART-
AF trial. J Am Coll Cardiol. 2014;64(7):647-56. https://doi.org/ 10.1016/j.jacc.2014.04.072.

16. Kuck KH, Brugada J, Furnkranz A, Metzner A, Ouyang F, Chun $\mathrm{KR}$, et al. Cryoballoon or radiofrequency ablation for paroxysmal atrial fibrillation. N Engl J Med. 2016;374(23):2235-45. https://doi. org/10.1056/NEJMoa1602014.

17. Dulac A, Sarrazin J, Nault I, O'Hara G, Philippon F, Molin F, et al. Comparison of pulmonary vein isolation using cryoballoon artic front advance versus contact force-guided radiofrequency for paroxysmal atrial fibrillation. Can J Cardiol. 2014;30:S287-8. https:// doi.org/10.1016/j.cjca.2014.07.512.13.

18. Osorio J, Hunter TD, Bubien RS, Rajendra A, Arciniegas J, Morales G. Gains in paroxysmal atrial fibrillation ablation using a standardized workflow to optimize contact force technologies. J Atr Fibrillation. 2018;11(4):2097. https://doi.org/10.4022/jafib.2097.

19. Squara F, Zhao A, Marijon E, Latcu DG, Providencia R, Di Giovanni G, et al. Comparison between radiofrequency with contact force-sensing and second-generation cryoballoon for paroxysmal atrial fibrillation catheter ablation: a multicentre European evaluation. Europace. 2015;17(5):718-24. https://doi.org/10.1093/ europace/euv060.

20. Padala SK, Gunda S, Sharma PS, Kang L, Koneru JN, Ellenbogen KA. Risk model for predicting complications in patients undergoing atrial fibrillation ablation. Heart Rhythm. 2017;14(9):1336-43. https://doi.org/10.1016/j.hrthm.2017.04.042.

21. Lip GY, Nieuwlaat R, Pisters R, Lane DA, Crijns HJ. Refining clinical risk stratification for predicting stroke and thromboembolism in atrial fibrillation using a novel risk factor-based approach: the euro heart survey on atrial fibrillation. Chest. 2010;137(2):26372. https://doi.org/10.1378/chest.09-1584.

22. Lip GY, Frison L, Halperin JL, Lane DA. Comparative validation of a novel risk score for predicting bleeding risk in anticoagulated patients with atrial fibrillation: the HAS-BLED (hypertension, abnormal renal/liver function, stroke, bleeding history or predisposition, labile INR, elderly, drugs/alcohol concomitantly) score. J Am Coll Cardiol. 2011;57(2):173-80. https://doi.org/10.1016/j.jacc. 2010.09.024.

23. Pisters R, Lane DA, Nieuwlaat R, de Vos CB, Crijns HJ, Lip GY. A novel user-friendly score (HAS-BLED) to assess 1-year risk of major bleeding in patients with atrial fibrillation: the euro heart survey. Chest. 2010;138(5):1093-100. https://doi.org/10.1378/ chest.10-0134.

Publisher's note Springer Nature remains neutral with regard to jurisdictional claims in published maps and institutional affiliations. 